\title{
A SOLUTION OF THE CONSERVATION LAW FORM OF THE SERRE EQUATIONS
}

\author{
C. ZOPPOU ${ }^{凶 1}$, S. G. ROBERTS ${ }^{1}$ and J. PITT ${ }^{1}$
}

(Received 16 February, 2015; accepted 19 June, 2015; first published online 28 March 2016)

\begin{abstract}
The nonlinear and weakly dispersive Serre equations contain higher-order dispersive terms. These include mixed spatial and temporal derivative flux terms which are difficult to handle numerically. These terms can be replaced by an alternative combination of equivalent temporal and spatial terms, so that the Serre equations can be written in conservation law form. The water depth and new conserved quantities are evolved using a second-order finite-volume scheme. The remaining primitive variable, the depthaveraged horizontal velocity, is obtained by solving a second-order elliptic equation using simple finite differences. Using an analytical solution and simulating the dambreak problem, the proposed scheme is shown to be accurate, simple to implement and stable for a range of problems, including flows with steep gradients. It is only slightly more computationally expensive than solving the shallow water wave equations.
\end{abstract}

2010 Mathematics subject classification: primary 76B15; secondary 35L65, 65M08.

Keywords and phrases: dispersive waves, conservation laws, Serre equations, finitevolume method.

\section{Introduction}

Dispersive waves can occur when the vertical fluid velocity has an influence on the behaviour of the flow and, therefore, cannot be ignored. This can occur when there is an abrupt change in fluid flow caused by an advancing front or when the fluid flows over changes in topography. For example, dispersive waves have been observed in the atmosphere with advancing cold fronts. Other examples include the trailing waves that accompany a tsunami or a tidal bore.

Two-dimensional systems of equations that include the influence of vertical velocity of the flow on the behaviour of the flow contain higher-order dispersive terms.

\footnotetext{
${ }^{1}$ Mathematical Sciences Institute, Australian National University, Canberra, ACT 2001, Australia; e-mail: Christopher.Zoppou@anu.edu.au,Stephen.Roberts@anu.edu.au,Jordan.Pitt@anu.edu.au. This is an Open Access article, distributed under the terms of the Creative Commons Attribution licence (http://creativecommons.org/licenses/by/4.0/), which permits unrestricted re-use, distribution, and reproduction in any medium, provided the original work is properly cited.

(c) Australian Mathematical Society 2016, Serial-fee code 1446-1811/2016\$16.00
} 
The Serre equations belong to this class of equations. They are highly nonlinear and weakly dispersive, and are based on the Euler equations describing the threedimensional motion of fluid particles in incompressible flow with constant density. By integrating the Euler equations over the water depth and assuming that the variation of the horizontal velocity components remains constant with water depth, the Serre equations can be derived. The Serre equations contain second- and higher-order dispersive terms including a mixed spatial and temporal term. Ignoring these terms results in the shallow water wave equations. The mixed spatial and temporal term complicates the solution of the Serre equations.

There are numerous schemes for solving the Serre equations [8, 11]. These schemes only consider smooth problems. We have developed a scheme that can handle both smooth and steep gradient flows by employing very efficient schemes for solving conservation laws. If the Serre equations can be written in conservation law form, then these schemes can be used to solve the Serre equations. There is potentially a significant saving in computational effort if the equations can be written in conservation law form. In addition, these schemes are capable of handling steep gradients in a problem.

The Serre equations can be written in conservation law form by replacing the mixed derivative dispersive term with a combination of temporal and spatial terms. This is described in this paper. The water depth and new conserved quantity can be evolved using the finite-volume method. The remaining primitive variable, the depth-averaged velocity, is obtained by solving a second-order elliptic equation using simple finite differences.

With this approach, the conserved quantities can be discontinuous, which can be handled efficiently by the finite-volume method and approximate Riemann solver, and it is only slightly more computationally expensive than solving the shallow water wave equations.

In Section 2, the Serre equations are given, as well as expressions for the vertical velocity and pressure distribution predicted by these equations. We then derive the conservation law form of the Serre equations in Section 3 and explain why we want to write them in this form. How we solve the conservation law form of the Serre equations is described in Section 4. Convergence results for the simulation of a soliton are used to validate the proposed model in Section 5, and the results for the simulation of the dam-break problem are provided in Section 6.

\section{One-dimensional Serre equations}

The Serre equations were first derived by Serre in 1953 [13] and later by Su and Gardner [15] and Seabra-Santos et al. [12], and are identical to the depth-averaged Green-Naghdi equations [5].

The Serre equations assume that there is no variation in the horizontal velocity in the vertical direction. Other assumptions can be made, such as a linear variation or a parabolic variation $[10,18]$. In these cases, additional dispersive terms are introduced into the equations. 
Using this assumption and integrating the Euler equations for incompressible fluid with constant density flowing over a fixed impermeable horizontal bed and satisfying certain kinematic and dynamic boundary conditions results in the Serre equations [12]

$$
\frac{\partial h}{\partial t}+\frac{\partial(\bar{u} h)}{\partial x}=0
$$

and

$$
\underbrace{\underbrace{\frac{\partial(\bar{u} h)}{\partial t}+\frac{\partial}{\partial x}\left(\bar{u}^{2} h+\frac{g h^{2}}{2}\right)}_{\text {Shallow water wave equations }}+\underbrace{\frac{\partial}{\partial x}\left(\frac{h^{3}}{3}\left[\frac{\partial \bar{u}}{\partial x} \frac{\partial \bar{u}}{\partial x}-\bar{u} \frac{\partial^{2} \bar{u}}{\partial x^{2}}-\frac{\partial^{2} \bar{u}}{\partial x \partial t}\right]\right)}_{\text {Dispersion terms }}=0}_{\text {Serre equations }}
$$

where $\bar{u}(x, t)$ is the depth-averaged velocity of the horizontal fluid velocity, $u(x, y, t)$, $h(x, t)$ is the water depth, $g$ is the acceleration due to gravity, $t$ is the time and $(x, y)$ is the horizontal and vertical coordinate system. The kinematic boundary conditions simply state that a fluid particle on the water surface or on the bed remains on that surface or bed and the only dynamic boundary condition is the atmospheric pressure at the water surface.

For the Serre equations, the vertical fluid velocity is a linear function of water depth, zero at the bed and a maximum of

$$
w(x, y, t)=\frac{\partial h}{\partial t}+\bar{u} \frac{\partial h}{\partial x}
$$

at the water surface. Assuming $w=0$ results in the nonlinear shallow water wave equations. by

The pressure distribution $p$ is the atmospheric pressure at the water surface, given

$$
p=p_{\mathrm{a}}+\rho g h+\frac{\rho h^{2}}{2}\left(\frac{\partial \bar{u}}{\partial x} \frac{\partial \bar{u}}{\partial x}-\bar{u} \frac{\partial^{2} \bar{u}}{\partial x^{2}}-\frac{\partial^{2} \bar{u}}{\partial x \partial t}\right)
$$

at the bed, where $p_{\mathrm{a}}$ is the atmospheric pressure at the water surface and $\rho$ is the density of water. It has a hydrostatic pressure term, $p_{\mathrm{a}}+\rho g h$, the shallow water wave assumption, which is modified by a term that accounts for changes in the flow.

The continuity equation (2.1a) is exact; no assumption has been made about the vertical velocity distribution for $u$. However, in the derivation of the momentum equation, the assumption of constant $u$ with water depth makes these equations weakly dispersive.

As highlighted in (2.1b), the Serre equations are the shallow water wave equations with additional higher-order terms. They include a third-order spatial term as well as a mixed spatial and temporal term, which is the last term in the 'Dispersion terms' of (2.1b). The mixed spatial and temporal term complicates the solution of the Serre equations. The momentum equation can also be rewritten in terms of the depth-averaged velocity. In this case, the momentum equation resembles a 
dispersion equation with a third-order spatial derivative term. Ignoring third-order space derivatives and product derivatives, the Serre equations become the Boussinesq equations [1].

\section{Conservation law form of the Serre equations}

We could solve the Serre equations using a variety of methods. However, if we can write the equations in conservation law form, then there are very efficient schemes for solving conservation laws, which can be used to solve the Serre equations. These schemes are capable of handling steep gradients in a problem and there is potentially a significant saving in computational effort if the equations can be written in conservation law form. Smaller time steps would be necessary, or a complicated implicit scheme would be required to solve the Serre equations in the form of equation (2.1).

For example, using a simple explicit finite-difference scheme, stability analysis of the finite-difference scheme for the advection equation (conservation law form) would show that the computational time step, $\Delta t$, is proportional to the computation distance step, $\Delta x$. For the diffusion equation the computational time step is proportional to $\Delta x^{2}$ and, for the dispersion equation, the time step is proportional to $\Delta x^{3}$. Potentially, there are considerable savings to be made if the Serre equations can be written in conservation law form, where $\Delta t \leq \Delta x$ would be the requirement.

By making the following observation:

$$
\frac{\partial}{\partial x}\left(\frac{h^{3}}{3} \frac{\partial^{2} \bar{u}}{\partial x \partial t}\right)=\frac{\partial}{\partial t}\left(h^{2} \frac{\partial h}{\partial x} \frac{\partial \bar{u}}{\partial x}+\frac{h^{3}}{3} \frac{\partial^{2} \bar{u}}{\partial x^{2}}\right)-\frac{\partial}{\partial x}\left(h^{2} \frac{\partial h}{\partial t} \frac{\partial \bar{u}}{\partial x}\right)
$$

and using the continuity equation to eliminate $\partial h / \partial t$, the momentum equation $(2.1 \mathrm{~b})$ can be rewritten, so that there are no temporal derivatives in the flux term, as

$$
\frac{\partial}{\partial t}\left(\bar{u} h-h^{2} \frac{\partial h}{\partial x} \frac{\partial \bar{u}}{\partial x}-\frac{h^{3}}{3} \frac{\partial^{2} \bar{u}}{\partial x^{2}}\right)+\frac{\partial}{\partial x}\left(\bar{u}^{2} h+\frac{g h^{2}}{2}-\bar{u} h^{2} \frac{\partial h}{\partial x} \frac{\partial \bar{u}}{\partial x}-\frac{\bar{u} h^{3}}{3} \frac{\partial^{2} \bar{u}}{\partial x^{2}}-\frac{2 h^{3}}{3} \frac{\partial \bar{u}}{\partial x} \frac{\partial \bar{u}}{\partial x}\right)=0 .
$$

The temporal derivative terms have a corresponding flux term. These can be combined to produce a new nonphysical conserved quantity

$$
G=\bar{u} h-h^{2} \frac{\partial h}{\partial x} \frac{\partial \bar{u}}{\partial x}-\frac{h^{3}}{3} \frac{\partial^{2} \bar{u}}{\partial x^{2}} .
$$

It consists of a flux of material $\bar{u} h$ which is modified by some function of the water surface profile and velocity. The momentum equation (2.1b) can be written in the desired conservation law form as

$$
\frac{\partial G}{\partial t}+\frac{\partial}{\partial x}\left(\bar{u} G+\frac{g h^{2}}{2}-\frac{2 h^{3}}{3} \frac{\partial \bar{u}}{\partial x} \frac{\partial \bar{u}}{\partial x}\right)=0 .
$$

The continuity equation (2.1a) is already in the appropriate form. 
Given $G$ and $h$, the remaining primitive variable $\bar{u}$ is calculated through the operator $\mathcal{A}[h, G]$, which is the solution of the second-order elliptic equation

$$
\bar{u} h-\frac{\partial}{\partial x}\left(\frac{h^{3}}{3} \frac{\partial \bar{u}}{\partial x}\right)=G,
$$

which is equation (3.1) written in divergent form and is easily solved using finite differences or the finite-element method.

\section{A finite-volume solution of the conservation law form of the Serre equations}

We solve the Serre equations using a second-order finite-volume method [17], where the cell interface values are obtained by using linear interpolations which are limited using the generalized minmod limiter [16] to prevent unwanted oscillations. This ensures that the results are physical (bounded) and, therefore, stable. It includes a parameter, $\theta \in[1,2]$, which controls the amount of diffusion introduced by the limiter.

Time integration of the semi-discrete system is performed using a second-order strong stability preserving (SSP) Runga-Kutta scheme [9, 14].

The numerical approximation of the physical flux of a conserved quantity $q_{j+1 / 2}^{ \pm}$ across the boundary of a cell is given by the approximate Riemann solver proposed by Kurganov et al. [7] as

$$
F_{j+1 / 2}=\frac{a_{j+1 / 2}^{+} f\left(q_{j+1 / 2}^{-}\right)-a_{j+1 / 2}^{-} f\left(q_{j+1 / 2}^{+}\right)}{a_{j+1 / 2}^{+}-a_{j+1 / 2}^{-}}+\frac{a_{j+1 / 2}^{+} a_{j+1 / 2}^{-}}{a_{j+1 / 2}^{+}-a_{j+1 / 2}^{-}}\left[q_{j+1 / 2}^{+}-q_{j+1 / 2}^{-}\right] .
$$

This only requires an estimate of the wave speeds, $a_{j+1 / 2}^{ \pm}$, and $f\left(q_{j+1 / 2}^{ \pm}\right)$, which is the corresponding finite-difference analogue of the flux function in (2.1a) or (3.2) for $q_{j+1 / 2}^{ \pm}$.

Performing a Fourier analysis of the linearized Serre equations, we find that the phase speed

$$
v_{\mathrm{p}}=u_{0} \pm \sqrt{g h_{0}} \sqrt{\frac{3}{k^{2} h^{2}+3}}
$$

differs from the group speed $v_{\mathrm{g}}$, which is a property of dispersive waves, where $u_{0}$ and $h_{0}$ are the unperturbed values for $u$ and $h$, respectively. As the wave number $k \rightarrow 0$, $v_{\mathrm{p}} \rightarrow v_{\mathrm{g}} \rightarrow u_{0} \pm \sqrt{g h_{0}}$; they are equal to the phase speed of shallow water waves, where all wave components travel at the same speed. Indeed, the phase speed for the Serre equations is bounded by the phase speed of the shallow water wave equations, that is,

$$
u_{0}-\sqrt{g h_{0}} \leq u_{0} \pm \sqrt{g h_{0}} \sqrt{\frac{3}{k^{2} h^{2}+3}} \leq u_{0}+\sqrt{g h_{0}} .
$$

We now have an estimate of the maximum and minimum wave speeds required by our chosen approximate Riemann solver. 
4.1. Solution process The solution of the Serre equations from the time step $n \Delta t$ to $(n+1) \Delta t$ involves the following steps:

$$
\begin{gathered}
\underbrace{\left[\begin{array}{l}
h \\
G
\end{array}\right]^{n} \stackrel{\mathcal{A}}{\rightarrow} \bar{u}^{n}}_{\text {I }} \rightarrow \underbrace{\left[\begin{array}{l}
h \\
G
\end{array}\right]^{(1)}=\left[\begin{array}{l}
h \\
G
\end{array}\right]^{n}-\Delta t \mathcal{L}\left[\begin{array}{l}
h \\
G
\end{array}\right]^{n}}_{\text {II First Euler step }} \\
\underbrace{\left[\begin{array}{l}
h \\
G
\end{array}\right]^{(1)} \stackrel{\mathcal{A}}{\longrightarrow} \bar{u}^{(1)}}_{\text {III }} \rightarrow \underbrace{\left[\begin{array}{l}
h \\
G
\end{array}\right]^{(2)}=\left[\begin{array}{l}
h \\
G
\end{array}\right]^{(1)}-\Delta t \mathcal{L}\left[\begin{array}{l}
h \\
G
\end{array}\right]^{(1)}}_{\text {IV Second Euler step }} \\
\underbrace{\left[\begin{array}{l}
h \\
G
\end{array}\right]^{n+1}}_{\text {V Averaging step }}=\frac{\frac{1}{2}\left[\begin{array}{l}
h \\
G
\end{array}\right]^{n}+\frac{1}{2}\left[\begin{array}{l}
h \\
G
\end{array}\right]^{(2)}}{}
\end{gathered}
$$

Step I. Given the conserved quantities $h$ and $G$, the remaining primitive variable $\bar{u}$ is obtained by solving the second-order elliptic equation (3.1) using finite differences.

Step II. Perform the reconstruction and solve a local Riemann problem to obtain the flux, $F_{j \pm 1 / 2}$, of material across a cell interface. Evolve the solution using a first-order Euler time integration for the conserved quantities.

Steps III and IV. Repeat the process with the intermediate values and evolve using another first-order Euler step.

Step $V$. The solution at the next time level is obtained by averaging the initial values and the values obtained from the second Euler step, which completes the second-order strong stability preserving Runge-Kutta time integration.

The second-order elliptic equation (3.1) is solved for $\bar{u}$ using second-order central differences. Equation (3.1) is approximated by

$$
G_{j}=a_{j} \bar{u}_{j+1}+b_{j} \bar{u}_{j}+c_{j} \bar{u}_{j-1},
$$

where $a_{j}=-h_{j}^{2}\left(h_{j+1}-h_{j-1}\right) /\left(4 \Delta x^{2}\right)-h_{j}^{3} /\left(3 \Delta x^{2}\right), \quad b_{j}=h_{j}+2 h_{j}^{3} /\left(3 \Delta x^{2}\right)$ and $c_{j}=$ $h_{j}^{2}\left(h_{j+1}-h_{j-1}\right) /\left(4 \Delta x^{2}\right)-h_{j}^{3} /\left(3 \Delta x^{2}\right)$ for the $m$ uniformly spaced computational nodes, $j=1, \ldots m$, which have been used to discretize the computational domain and $G_{j}$ and the corresponding water depth at each node, $h_{j}$, are obtained by solving the Serre equations written in conservation law form. This results in a tri-diagonal system of equations, which can be solved efficiently using direct methods for $\bar{u}_{j}$, given $G_{j}$ and $h_{j}$.

With this approach $h$ and $G$ can be discontinuous, which is handled by the finitevolume method and approximate Riemann solver efficiently. An attractive feature of this approach is that even if $G$ is discontinuous, $\bar{u}$ will always be smooth.

The resulting numerical scheme is theoretically $O\left(\Delta x^{2}, \Delta t^{2}\right)$ accurate. Theoretically, stability is satisfied when the time step, $\Delta t$, satisfies the Courant-Friedrichs-Lewy $(C F L)$ criterion [6]

$$
\Delta t<\frac{\Delta x}{2 \max \left(\left|\lambda_{i}\right|\right)} \quad \text { for all } i
$$




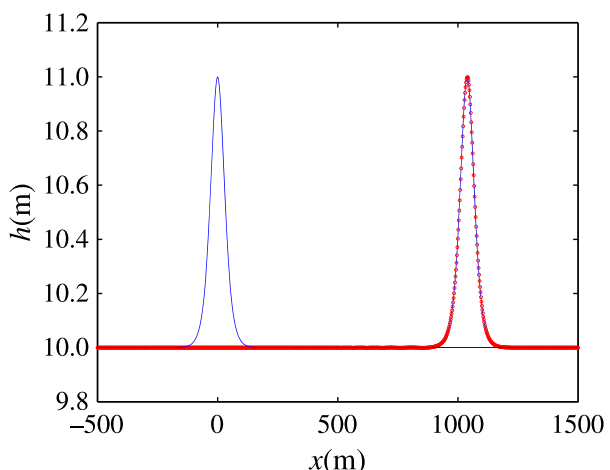

(a)

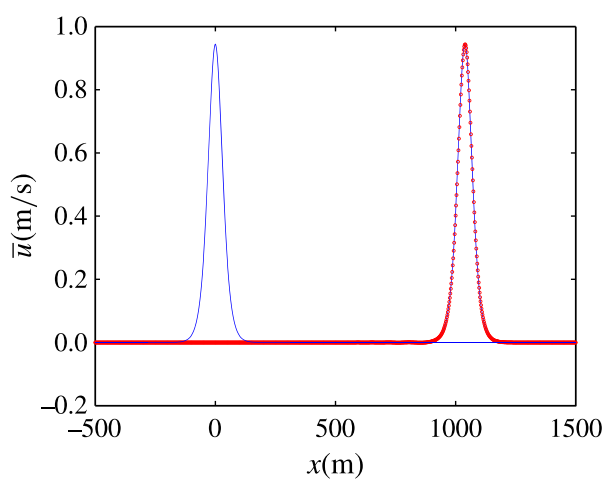

(b)

FIGURE 1. Initial and analytical solution of the Serre equations (blue line - ) plotted against the simulated solution of the Serre equations written in conservation law form (red circles $\circ$ ) with $h$ shown in $(a)$ and $\bar{u}$ in (b) (colour available online).

\section{Simulation of a soliton}

The only known analytical solution to the Serre equations is the Rayleigh solitary wave $[2,3]$, where

$$
h(x, t)=a_{0}+a_{1} \operatorname{sech}^{2}(\kappa(x-c t))
$$

and

$$
\bar{u}(x, t)=c\left(\frac{h(x, t)-a_{0}}{h(x, t)}\right)
$$

with $\kappa=\sqrt{3 a_{1}} /\left(2 a_{0} \sqrt{a_{0}+a_{1}}\right)$ and $c=\sqrt{g\left(a_{0}+a_{1}\right)}$, which is a soliton advected over a horizontal bed. The soliton shown in Figure 1 has an amplitude of $a_{1}=1.0 \mathrm{~m}$ and moves at a constant speed $c=10.387974 \mathrm{~m} \mathrm{~s}^{-1}$ without changing shape in the water that is $a_{0}=10 \mathrm{~m}$ deep. It represents a balance between the dispersive and nonlinear terms. If there is an imbalance between the nonlinear and dispersive terms, we would observe trailing waves as well as attenuation of the simulated profile. The analytical solution is shown as the solid blue line and the red dots as the simulated results, using our model with $\Delta x=2 \mathrm{~m}, \Delta t=0.2 \Delta x / \sqrt{g a_{0}} \mathrm{~s}, \theta=1.2$, and the solution is terminated at $t=100 \mathrm{~s}$. There is very little phase error and attenuation in the simulated results.

We have established the convergence rate for the model using the soliton problem by calculating the nondimensionalized $L_{1}$ norm

$$
L_{1}=\frac{\sum_{j=1}^{m}\left|h_{j}-h\left(x_{j}\right)\right|}{\sum_{j=1}^{m}\left|h\left(x_{j}\right)\right|},
$$




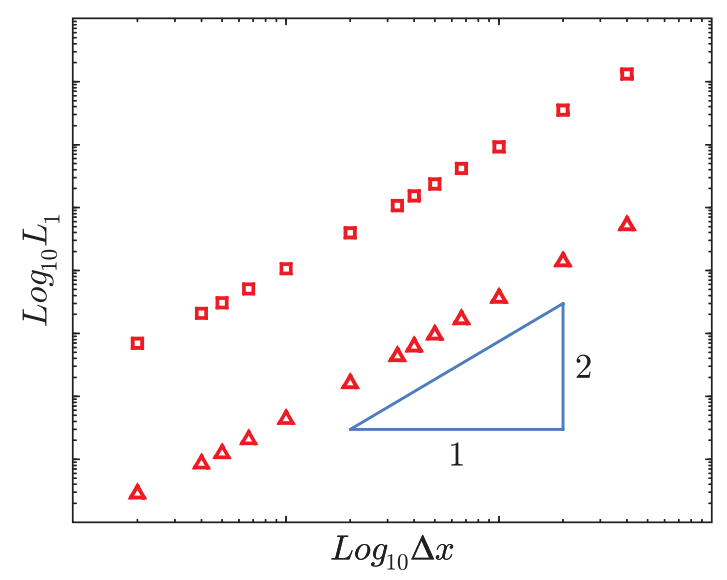

FIGURE 2. Convergence rate for the simulation of the soliton problem using the Serre equations written in conservation law form with water depth (triangles $\triangle$ ) and velocity (squares $\square$ ).

which is shown here for $h$ with $h_{j}$ as the simulated values of $h(x, t)$ at $x_{j}$ and $h\left(x_{j}\right)$ as the corresponding analytical solution. The $L_{1}$ norm is calculated using all the computational nodes. Figure 2 shows that the model is accurate up to the second order.

\section{Dam-break problem}

Initially, the water is still and $h_{1}=10 \mathrm{~m}$ deep to the right and $h_{0}=2 \mathrm{~m}$ deep to the left of a hypothetical dam on a frictionless horizontal channel. The solution is sought at $t=30 \mathrm{~s}$ after the dam is suddenly removed. In the simulations, $\Delta x=0.02 \mathrm{~m}$, $m=50000, \Delta t=0.2 \Delta x / \sqrt{g h_{1}}$ s and $\theta=1.2$. The results shown in Figure 3(a) are for the shallow water wave equations, where dispersion is ignored and in Figure $3(b)$ for the solution of the Serre equations written in conservation law form. We have also plotted the analytical solution to this problem for the shallow water wave equations, which is shown by the solid blue line.

There are no dispersive waves predicted by the shallow water wave equations. The Serre equations have produced oscillations in the solution. It has also accurately captured the shock and rarefaction fan.

The results shown in Figure 3 are similar to those obtained by El et al. [4], who used a finite-difference scheme to solve the Serre equations.

Due to the additional solution of the second-order elliptic equation, the scheme is approximately $60 \%$ more computationally expensive than solving the shallow water wave equations.

\section{Conclusions}

The Serre equations contain dispersive terms which account for the effects of vertical velocity of the fluid particles on the behaviour of the flow. They contain 


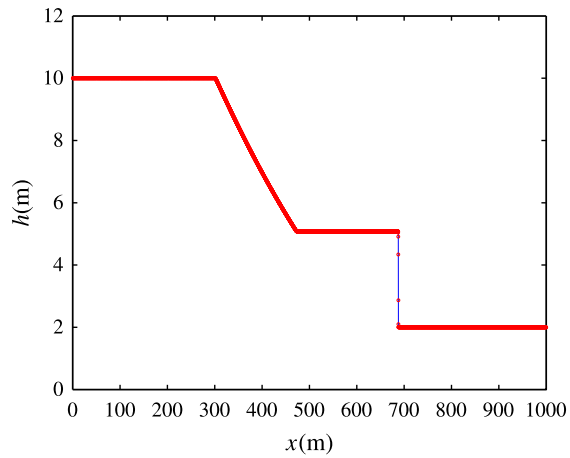

(a)

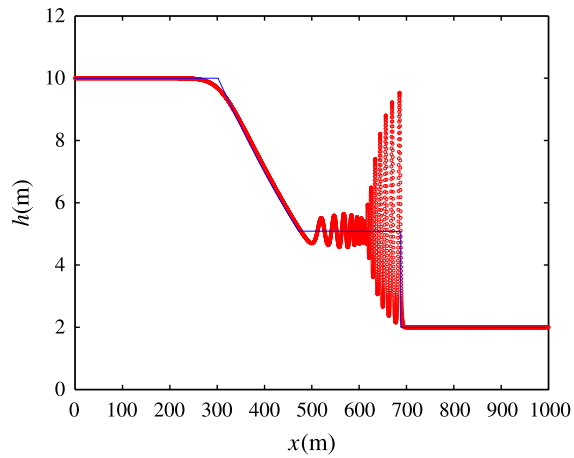

(b)

FIGURE 3. The simulated (red circles $\circ$ ) water depth, $h$, for the dam-break problem using the $(a)$ shallow water wave equations and $(b)$ Serre equations written in conservation law form shown along with the analytical solution (solid blue line - ) of the shallow water wave equations for this problem (colour available online).

higher-order spatial terms and a mixed spatial and temporal derivative term. The Serre equations have been written in conservation law form by replacing the mixed spatial and temporal derivative terms in the momentum equation by temporal terms and their corresponding fluxes. The temporal terms are combined to produce a new conservative quantity. The second-order finite-volume method is used to evolve the conserved quantities. The remaining primitive variable is obtained by solving a second-order elliptic equation. The attractive features of this approach are that steep gradients in the flow can be handled; it is more accurate and stable than the use of a finite-difference scheme and is only slightly more computationally expensive than solving the shallow water wave equations. This is demonstrated by simulating a hypothetical dam-break problem.

\section{Acknowledgement}

The work undertaken by the first author was supported financially by an Australian National University Postgraduate Research Award.

\section{References}

[1] D. R. Basco, "Computation of rapidly varied, unsteady, free-surface flow", Water-Resources Investigations Report 83-4284, US Geological Survey, 1987; http://pubs.usgs.gov/wri/1983/4284/report.pdf.

[2] J. D. Carter and R. Cienfuegos, "Solitary and cnoidal wave solutions of the Serre equations and their stability", Eur. J. Mech. B 30 (2011) 259-268; doi:10.1016/j.euromechflu.2010.12.002.

[3] F. Chazel, D. Lannes and F. Marche, "Numerical simulation of strongly non-linear and dispersive waves using a Green-Naghdi model", J. Sci. Comput. 48 (2011) 105-116; doi:10.1007/s10915-010-9395-9. 
[4] G. A. El, R. H. J. Grimshaw and N. F. Smyth, "Asymptotic description of solitary wave trains in fully nonlinear shallow-water theory", Physica D 237 (2008) 2423-2435; doi:10.1016/j.physd.2008.03.031.

[5] A. E. Green and P. M. Naghdi, "A derivation of equations for wave propagation in water of variable depth", J. Fluid Mech. 78 (1976) 237-246; doi:10.1017/S0022112076002425.

[6] A. Harten, "High resolution schemes for hyperbolic conservation laws", J. Comput. Phys. 49 (1983) 357-393; http://arrow.utias.utoronto.ca/ groth/aer1319/Handouts/Additional_Reading _Material/JCP-1983-harten.pdf.

[7] A. Kurganov, S. Noelle and G. Petrova, "Semidiscrete central-upwind schemes for hyperbolic conservation laws and Hamilton-Jacobi equations", J. Sci. Comput. 23 (2002) 707-740; doi:10.1137/S1064827500373413.

[8] M. Li, P. Guyenne, F. Li and L. Xu, "High order well-balanced CDG-FE methods for shallow water waves by a Green-Naghdi model", J. Comput. Phys. 257 (2014) 169-192; doi:10.1016/j.jcp.2013.09.050.

[9] C. B. Macdonald, S. Gottlieb and S. J. Ruuth, "A numerical study of diagonally split Runga-Kutta methods for PDEs with discontinuities", J. Sci. Comput. 6 (2008) 89-112; doi:10.1007/s10915-007-9180-6.

[10] P. A. Madsen and O. R. Sørensen, "A new form of Boussinesq equations with improved linear dispersion characteristics. Part II. A slowly-varying bathymetry", Coast. Eng. 18 (1992) 183-204; doi:10.1016/0378-3839(92)90019-Q.

[11] N. Panda, C. Dawson, Y. Zhang, A. B. Kennedy, J. J. Westerink and A. S. Donahue, "Discontinuous Galerkin methods for solving Boussinesq-Green-Naghdi equations in resolving non-linear and dispersive surface water waves", J. Comput. Phys. 273 (2014) 572-588; doi:10.1016/j.jcp.2014.05.035.

[12] F. J. Seabra-Santos, D. P. Renouard and A. M. Temperville, "Numerical and experimental study of the transformation of a solitary wave over a shelf or isolated obstacle", J. Fluid Mech. 176 (1987) 117-134; doi:10.1017/S0022112087000594.

[13] F. Serre, "Contribution à l'étude des écoulements permanents et variables dans les canaux", La Houille Blanche 6 (1953) 830-872; doi:10.1051/lhb/1953058.

[14] C. W. Shu and S. Osher, "Efficient implementation of essentially non-oscillatory shock-capturing schemes", J. Comput. Phys. 77 (1988) 439-471; doi:10.1016/0021-9991(88)90177-5.

[15] C. H. Su and C. S. Gardner, "Korteweg-de Vries equation and generalisations. III. Derivation of the Korteweg-de Vries equation and Burgers equation", J. Math. Phys. 10 (1969) 536-539; doi:10.1063/1.1664873.

[16] B. van Leer, "Towards the ultimate conservative difference scheme, V. A second-order sequel to Godunov's method", J. Comput. Phys. 32 (1979) 101-136; doi:10.1016/0021-9991(79)90145-1.

[17] C. Zoppou and S. Roberts, "Catastrophic collapse of water supply reservoirs in urban areas", J. Hydraul. Eng. 125 (1999) 11-34; doi:10.1061/(ASCE)0733-9429(1999)125:7(686).

[18] Z. L. Zou, "Higher order Boussinesq equations", Ocean Eng. 26 (1999) 767-792; doi:10.1016/S0029-8018(98)00019-5. 